\title{
Microscopic Images Restoration of Chinese Herbal Medicine Using the TV Model
}

\author{
Liu Qing $^{*}, 1$, Zhang Lijun ${ }^{1}$, Yang Xiaoping ${ }^{1}$ and Liu Xiping ${ }^{2}$ \\ ${ }^{I}$ School of Electronic Information \& Electrical Engineering, Tianshui Normal University, Tianshui, Gansu, 741001, \\ P.R. China \\ ${ }^{2}$ Department of Basic Medical Science Gansu University of Traditional Chinese Medicine, Gansu,Lanzhou, 730000, P.R. \\ China
}

\begin{abstract}
Chinese herbal medicine (CHM) samples defiled, extraction of microscopic images, collection site light not equal and images collected uneven illumination point which can make the acquisition of CHM microscopic images subject to different unfavorable factors. Those often make microscopic images of CHM become blurred or introduce noise. In this paper, some restoration methods for the microscopic images of CHM using the total variation (TV) model are put forward. Firstly, the microscopic images of CHM are blurred utilizing the two dimensional different Gaussian convolution kernels and different Gauss white noise are added, and then the damaged images are restored by using the improved TV model. The TV restoration results are compared with the results using Wiener filtering, median filtering method etc. The theoretical analysis and experimental results show that the novel method is effectively able to restore image, and maintain the image detail information.
\end{abstract}

Keywords: Images restoration, microscopic image of CHM, noise removal, TV model.

\section{INTRODUCTION}

Chinese Herbal Medicine (CHM) is the traditional national industry in China, a novel type of industry and the unique important component of Chinese medicine economy rapidly developed in $21^{\text {st }}$ century. This brings various good opportunities to the traditional Chinese medicine with all sorts of variation in modern domestic and international medical market. The trend of the development of medical market today, also form the severe transformation. The fake shoddy, degradation and other factors of CHM can affect the clinical curative effect and its quality, so distinction, identification and control of Chinese herbal medicine are very important research topics in the modern medical field. The traditional CHM distinction mainly relies on subjective experience to judge, which can cause a lot of false recognition rate. Modern chemical and physical identification of CHM utilize specialized test and analysis equipments. These will lead to the high cost of testing and the complex operations [1]. The testing and identification of $\mathrm{CHM}$ using the modern information technology is the new direction [2]. The modern identification, testing and recognition of CHM based on the technology of the image signal processing are one of the advanced methods used in the IT field.

Image restoration is an important role of the image processing. The purpose of image restoration is to improve the

\footnotetext{
*Address correspondence to this author at the School of Electronic Information \& Electrical Engineering, Tianshui Normal University, Tianshui, Gansu, 741001, P.R. China; Tel: 0938-8366657; E-mail: xdlq@163.com
}

image quality. The observed image is usually the degradation image under factors of the noise, fuzzy, sports translation [3]. The classic image restoration process is based on prior knowledge to build an image degradation model, and then a variety of the degradation processing methods are adopted to make the image quality improved. From the maintained image edge structures, this paper uses the idea of regularization to restore $\mathrm{CHM}$ image. The sample carrying equipment, prolonged exposure and movement of the microscopy devices can make motion blur, atmospheric or defocusing motion, and processing of the noise, would cause the degradation of the microscopic image in the process acquisition. Therefore, the image degradation should be recovered before understanding and analysis of image. Firstly, the CHM microscopic images are obtained utilizing the microscope, and then the total variation (TV) model [4] is used in the image restoration processing.

\section{THE TV MODEL OF IMAGE RESTORATION}

\subsection{Image Restoration Problem Description}

Image restoration refers to that the original of the ideal image is restored based on the recorded degraded images and a prior knowledge of degraded system. The key of the image restoration processing is to establish the model of degradation and restoration $[5,6]$.

Fig. (1) shows the block diagram of image. Image restoration is a process of prediction and estimation, the system parameter $H$ will be estimated from the given degraded 


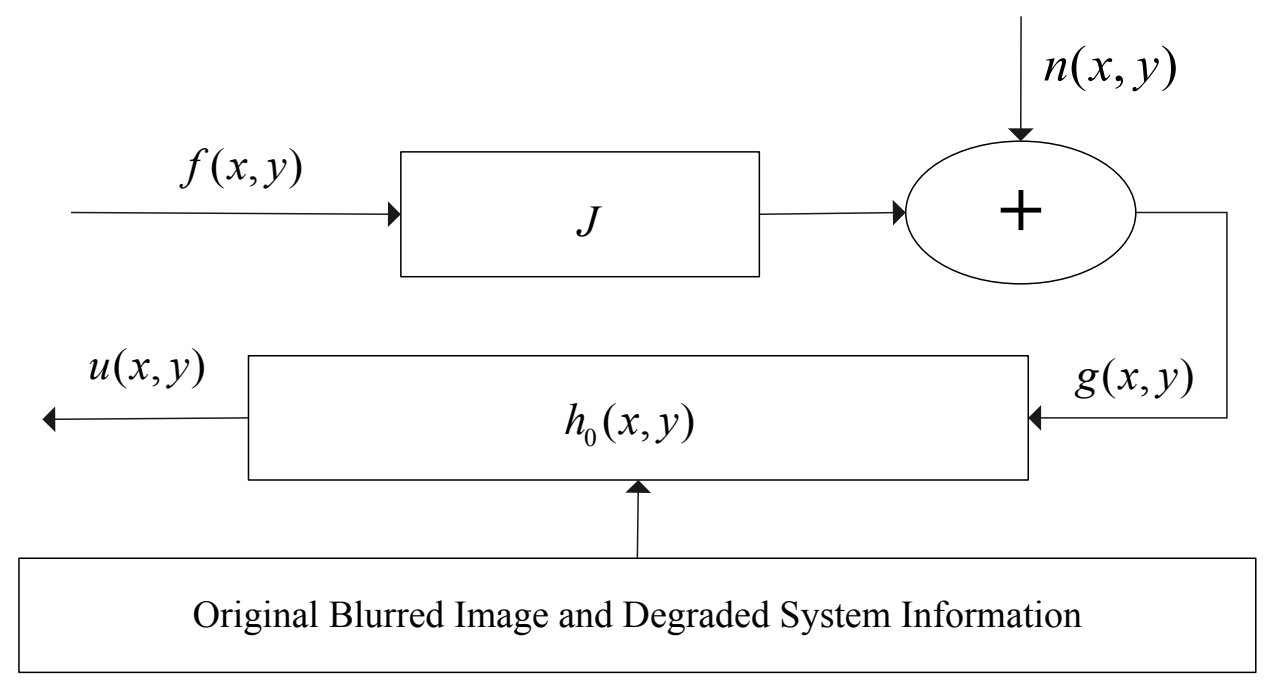

Fig. (1). Image degradation and restoration.

image $g(x, y)$, and then $f(x, y)$ will be approximately restored. That is the original image $f(x, y)$ effect by the point spread function (PSF) of degraded image model, the degraded system $J$, and then the result of the additive noise function $n(x, y)$ is the sum of the image $g(x, y)$, the $u(x, y)$ is the estimated value of the original image which is estimated using image restoration algorithm $h_{0}(x, y)$ through the original polluted image and portion PSF information, it approaches the possibility of the clear original image.

The mathematical expression of the degradation image model can be written:

$g(x, y)=f(x, y) * J+n(x, y)$

$g(x, y)$ is a degraded image, $f(x, y)$ is an original image, $h_{0}(x, y)$ is the PSF of the image degradation model, $n(x, y)$ is not related to noise and image, "** denotes the convolution in expression (1).

The Fourier transform form of the image degradation model can be expressed as follows:

$G(u, v)=F(u, v) J(u, v)+N(u, v)$

Where $G(u, v), F(u, v), J(u, v)$ and $N(u, v)$ are $g(x, y)$, $f(x, y), J(x, y)$ and $n(x, y)$ frequency domain representation respectively. The point spread function $J(x, y)$ is additive, consistent, linear and position invariant in a degraded system. Therefore, at present there are some image restoration methods, such as inverse filtering, Weiener and least square. The starting point of wiener deconvolution filter theory is to make the output image and the ideal image of the minimum mean square error (MSE) [7]; it can be expressed as follows:

$$
E\{h\}=E\left\{(f-u)^{2}\right\}=E\left\{(f-h *(J * f+n))^{2}\right\}
$$

And the least square method constitutes as a functional variation problem, and makes the equation (4) minimum.

$E(u)=\iint_{\Omega}|\nabla u|^{2} d \Omega+\lambda \iint_{\Omega}\left|h_{d} * u-u_{0}\right|^{2} d \Omega$

$\lambda$ is Lagrange multiplier.

There is some variation in the above methods and traditional image restoration method. On the whole, the effect is often not satisfactory. Especially for the strong noise, obvious ringing, residual noise and blur is retained in the output image.

\subsection{Image Restoration Problem Description}

Actually, the abrupt change or the edge of an image is its inherent characteristics. The punishment for large gradient will be particularly emphasized if using $\int|\nabla u|^{2}$ as measure of smoothness. Rudin, Osher and Fatime [8] first proposed the measure of image smoothness with $\int|\nabla u|$ based on the above factors, which created a new image restoration method, the total variation image restoration method.

When using $|\nabla u|$ instead of $|\nabla u|^{2}$ in equation (4), the energy functional of image restoration is generated based on TV.

$$
E(u)=\int|\nabla u| d \Omega+\lambda \int\left[h_{d} * u-u_{0}\right]^{2} d \Omega
$$

The model of the total variation image restoration is formed using the Euler-Lagrange equation; it can be expressed as follows:

$\lambda h_{d} *\left(h_{d} * u-u_{0}\right)-\operatorname{div}\left(\frac{\nabla u}{|\nabla u|}\right)=0$

Equation (6) would result in the ill-conditioned PDE because the pixel points with $\nabla u=0$ exist in the image. Equation (6) can be regularized if a small integer $\alpha$ is introduced 
in the total variation, the TV can be written as the following form:

$$
\operatorname{TV}(u)=\iint_{\Omega}|\nabla u|_{\alpha} d \Omega=\iint_{\Omega} \sqrt{u_{x}^{2}+u_{y}^{2}+\alpha} d x d y, \quad \alpha>0
$$

Then

$\lambda h_{d} *\left(h_{d} * u-u_{0}\right)-\operatorname{div}\left(\frac{\nabla u}{|\nabla u|_{\alpha}}\right)=0$

Solution of this equation by the gradient descent method,

$$
\frac{\partial u}{\partial t}=\operatorname{div}\left(\frac{\nabla u}{|\nabla u|_{\alpha}}\right)-\lambda h_{d} *\left(h_{d} * u-u_{0}\right)
$$

Solution of the $\mathrm{u}$ is finally restored image.

TV the iterative restoration equations are a kind of anisotropic diffusion process. In fact, the slower speed of iteration should be used in the image features' obvious region, and iterative restoration speed should be accelerated in the region where the image characteristic is not obvious. But the diffusion efficiency is low when the single anisotropic diffusion process is used in TV model [9]. In addition, the factor $\operatorname{div}(\nabla u /|\nabla u|)$ can be decomposed into only to spread in the direction of gradient orthogonal $\nabla u$ under the local coordinate system. Therefore, TV model diffuses all along the edge direction on the whole image, which shows different ideas along the direction of the different diffusion coefficient. Usually, the edge direction is not real in flat areas of the image. It would lead to insufficient noise suppression, even the false edge, and the staircase effect in the plain area will still spread only in the edge direction.

In fact, the diffusion velocity of TV restoration model is larger, but it is still only along with the direction of the gradient orthogonal spread when the pixel change is small in the $(x, y)$ neighborhood. In this case, the $(x, y)$ neighborhood does not contain complex image information; fully isotropic heat diffusion equation can be used.

In summary, a combination of the anisotropic and the isotropic nonlinear diffusion equation is put forward, which can use different restoration equation in different regions.

$$
\begin{cases}\frac{\partial u}{\partial t}=\frac{1}{G_{\nabla}}(\Delta u) & G_{\nabla}<\mathrm{K} \\ \frac{\partial u}{\partial t}=\frac{1}{G_{\nabla}}\left(\operatorname{div}\left(\frac{\nabla u}{|\nabla u|_{\alpha}}\right)-\lambda h_{d} *\left(h_{d} * u-u_{0}\right)\right), G_{\nabla} \geq \mathrm{K}\end{cases}
$$

$G_{\nabla}=\sqrt{u_{x}^{2}+u_{y}^{2}}$ is the image gradient modulus value at point $(x, y)$, and $\mathrm{K}$ is the image restoration threshold, which can be set according to different images.

The solution of this model can make sure the edges of the image are maintained, and also speed up to restore image in the smooth area.

\section{CALCULATION METHOD AND PROCESS}

The diffusion operator $\operatorname{div}(\nabla u /|\nabla u|)$ is discretized using any difference method. Then $u=\left(u_{1}, u_{2}\right)=(\nabla u /|\nabla u|)$, its divergence is approximation for:

$\nabla u=\frac{\partial u_{1}}{\partial x}+\frac{\partial u_{2}}{\partial y}$

Then equation (10) is simplified using Gauss-Jacobi iteration algorithm.

On image diffusion iterations, firstly, a threshold is taken and the old pixels are substituted by the new calculated pixel values. When the difference of the old and the new image pixel values is less than the given threshold, then iteration is stopped. Then it is applied to a new pixel values instead of corresponding pixels, until all the pixels are processed. Finally, the image restoration is finished.

The calculation of image restoration process is as follows:

(1) The contaminated image is read and normalized.

(2) The eight neighborhood of the reference point is selected; the pixel values and its gradient value from that point are calculated. Then the corresponding iterative equation is selected by comparing with the threshold value.

(3) The new pixel value is calculated according to the simplified equation (10), and the value of pixel value instead of the original image.

(4) The difference between the two iterations of pixel values before and after is determined. The iteration is stopped if the difference is less than a fixed value, or continues to iterate.

\section{EXPERIMENTAL RESULTS AND ANALYSIS}

Many different Chinese traditional medicine microscopic images are blurred by two dimensional Gaussian convolutions kernels and added Gauss white noise. In this experiment, section diagram of Radices sileris, organization chart of Fructus galangae, skin cells figure of Schisandra chinensis and many other different microscopic images of Chinese herbal medicine were blurred by two dimensional Gaussian convolution kernels, polluted by different Gauss white noise, and then restored using the TV model. The chosen parameters $\Delta t=0.1, \lambda=1.5$ of the TV model were used in the experiment. At last, the experimental results were compared with the other filters: (a) is the original image, (b) is the image blurred by the different convolution and Gauss noise variance, (c) is the restored image using the median filtering, (d) is wiener filtering restored image and (e) is restored image using the proposed TV algorithm in Fig. (2), Fig. (3) and Fig. (4).

From the experimental results it can be seen that the median filtering image to the convolution is blurring due to 


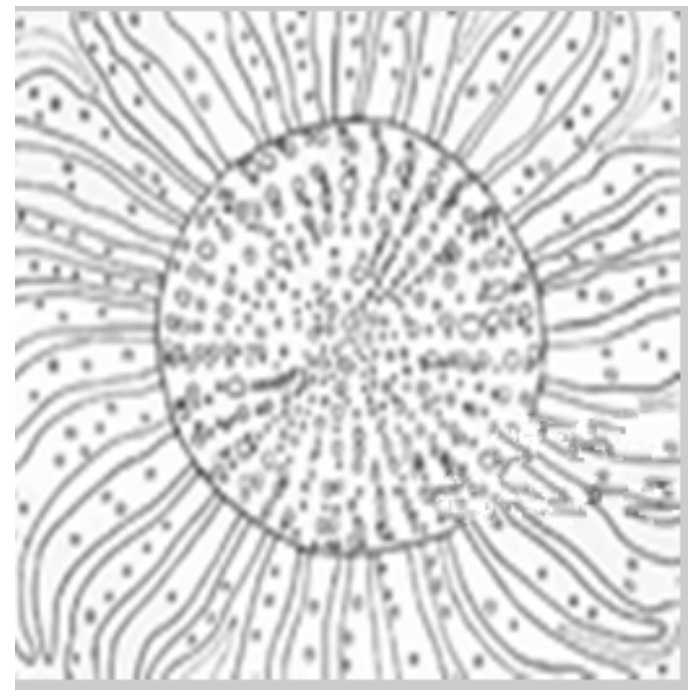

(a)

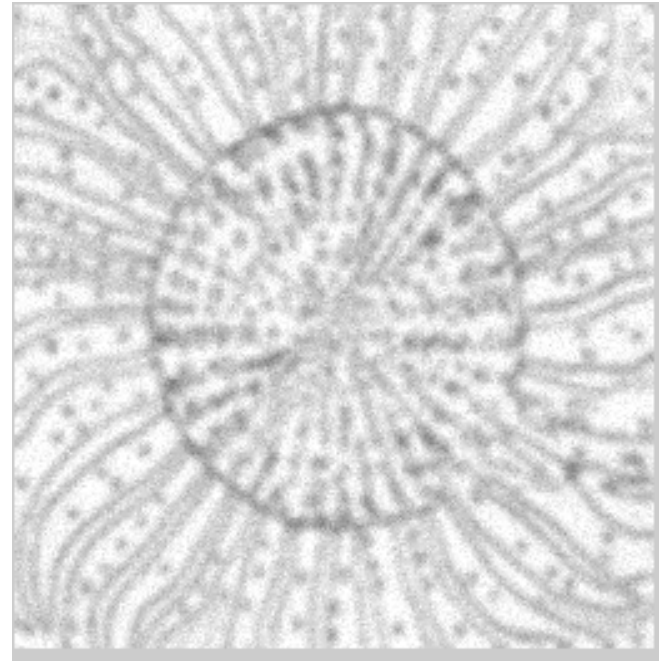

(b)

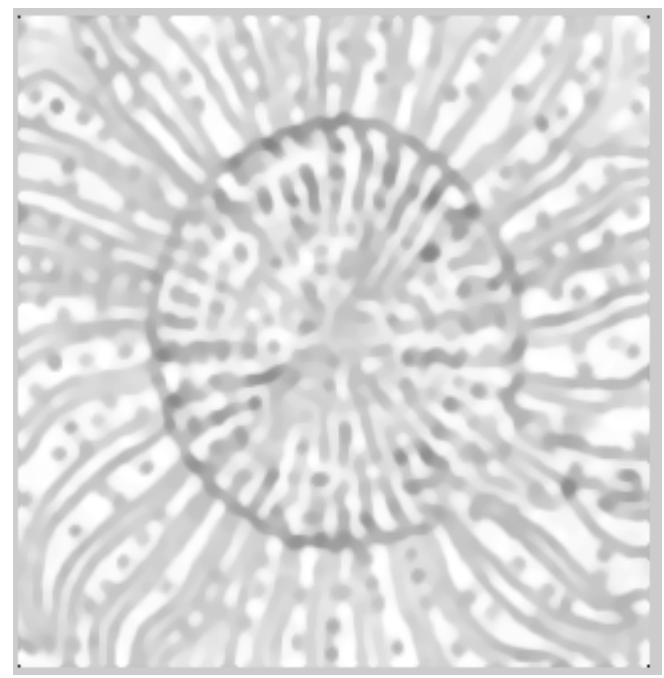

(c)

Fig. (2). Contd... 


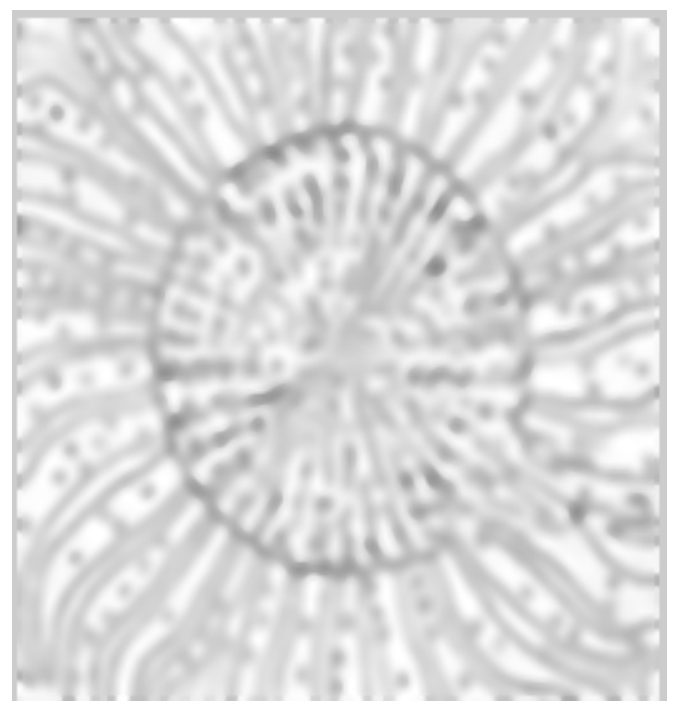

(d)

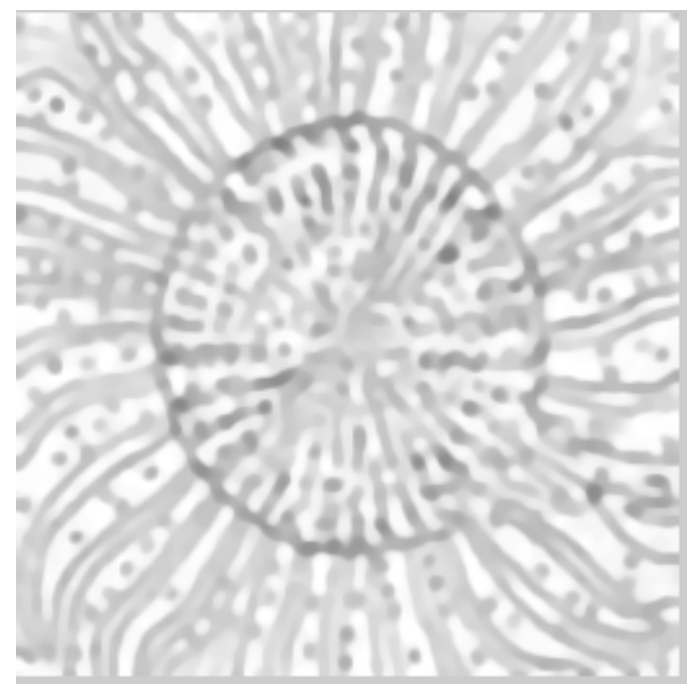

(e)

Fig. (2). Restoration results of different algorithms to Radices sileris microscopic image affected by the different blurred convolution kernels and the noise variance. (a) Original image; (b) The image is blurred by the convolution kernels for 7 and the noise variance for 3; (c) Median filtering; (d) Wiener filtering; (e) TV model restoration.

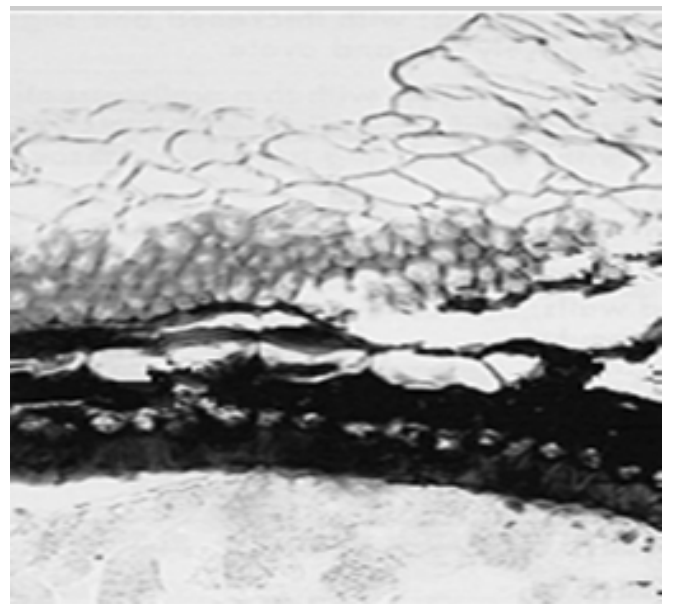

(a)

Fig. (3). Contd... 


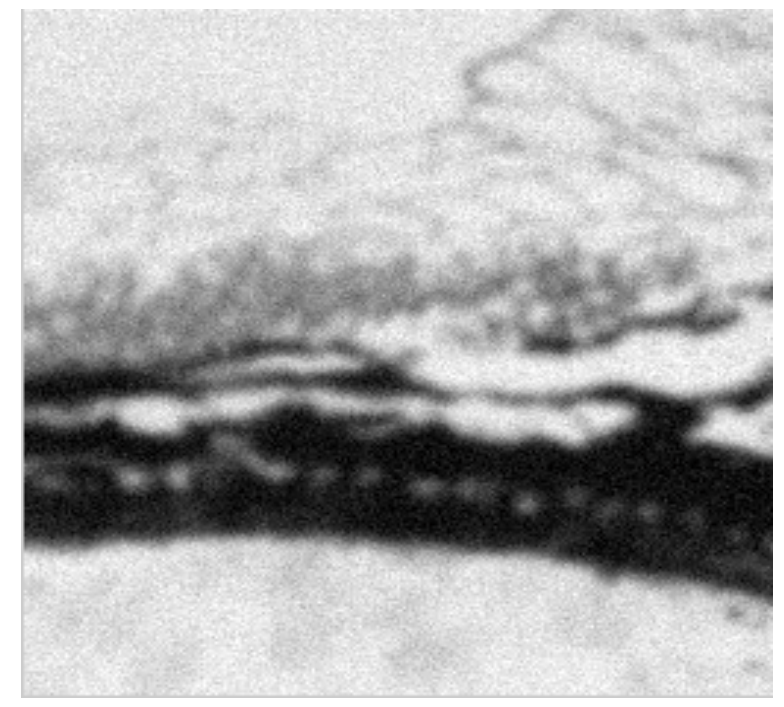

(b)

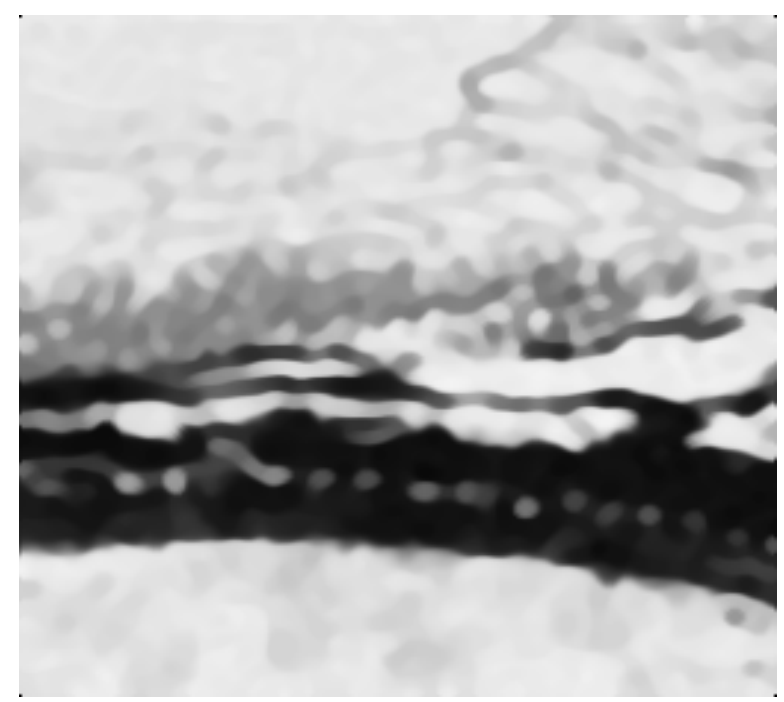

(c)

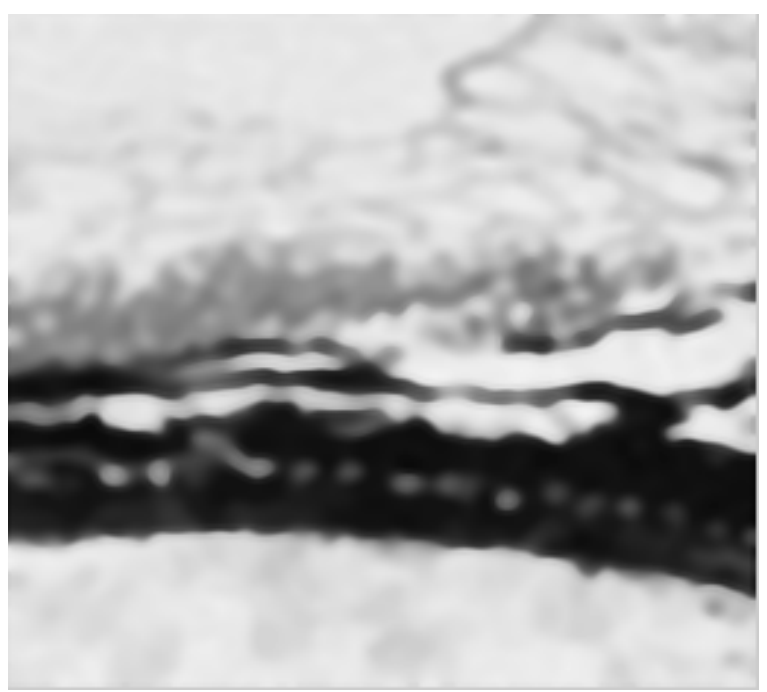

(d)

Fig. (3). Contd... 


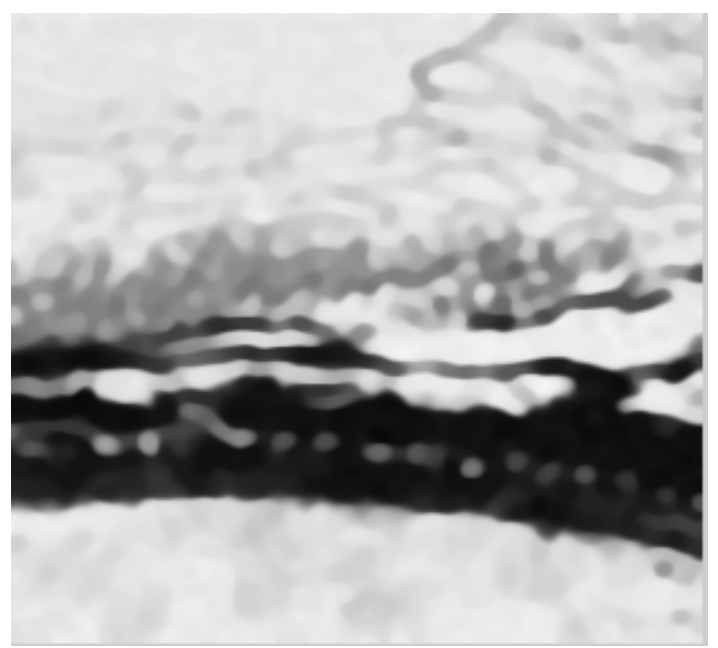

(e)

Fig. (3). Restoration results of different algorithms to Fructus galangae microscopic image affected by the different blurred convolution kernels and the noise variance. (a) Original image; (b) The image is blurred by the convolution kernels for 7 and the noise variance for 3; (c) Median filtering; (d) Wiener filtering; (e) TV model restoration.

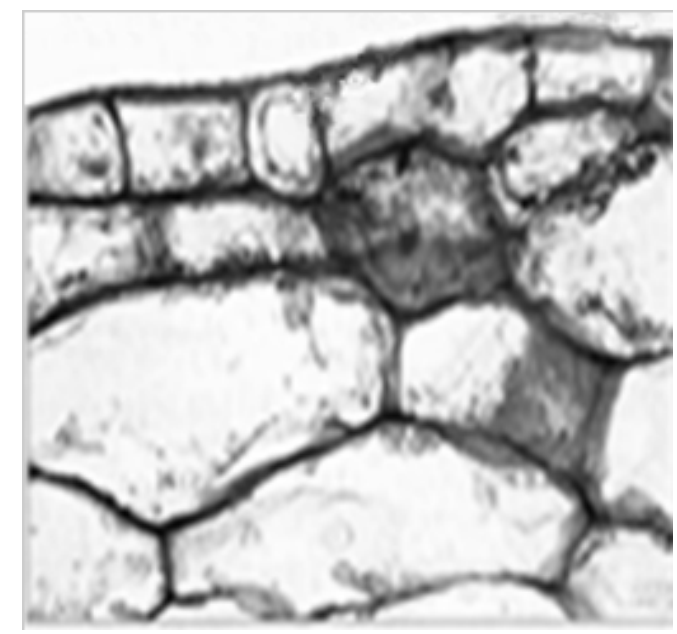

(a)

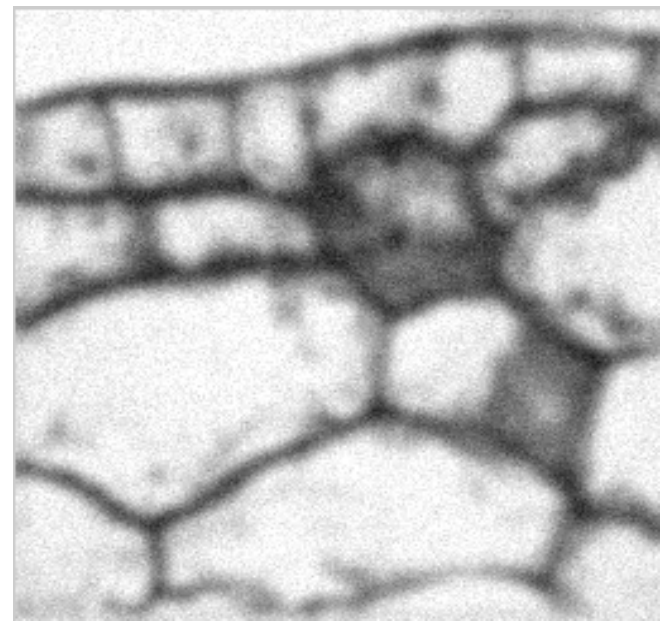

(b)

Fig. (4). Contd... 


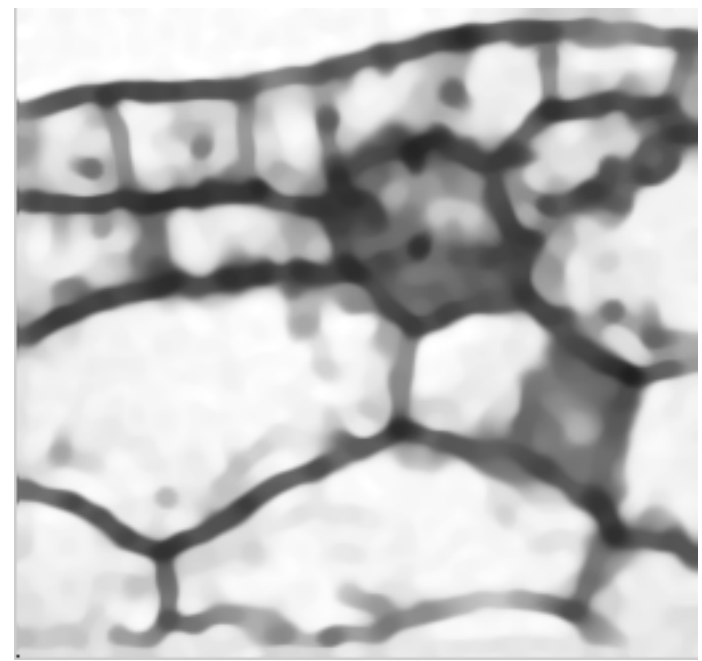

(c)

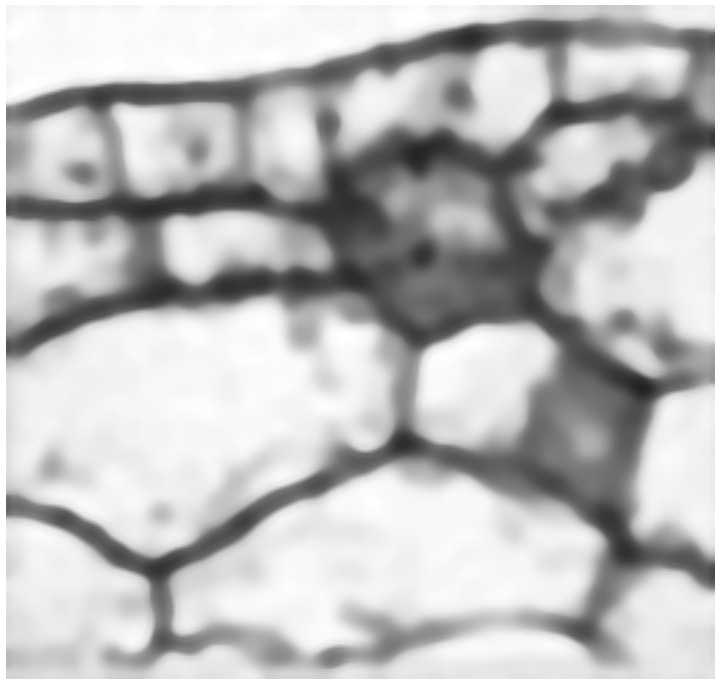

(d)

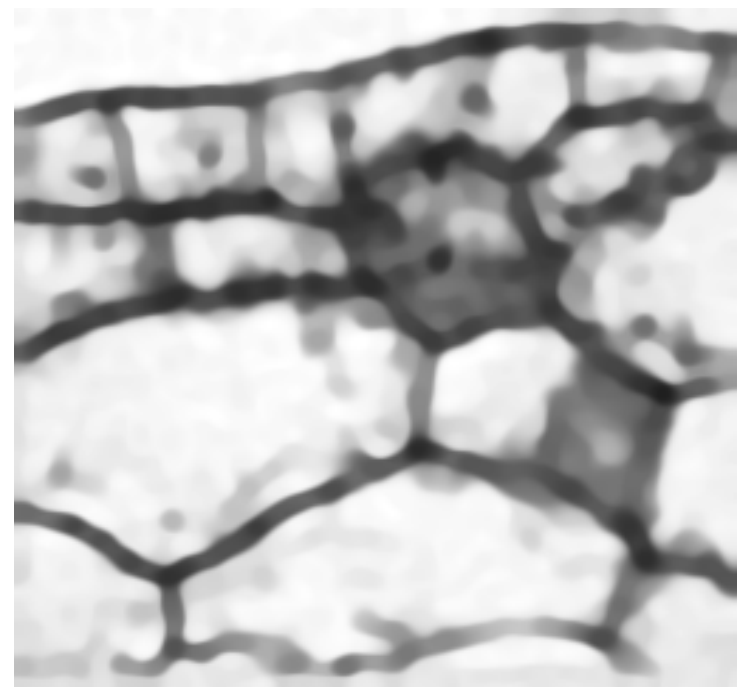

(e)

Fig. (4). Restoration results of different algorithms to Schisandra chinensis microscopic image affected by the different blurred convolution kernels and the noise variance. (a) Original image; (b) The image is blurred by the convolution kernels for 7 and the noise variance for 3 ; (c) Median filtering; (d) Wiener filtering; (e) TV model restoration. 
Gauss white noise pollution. Although the noise filtering capability is strong, yet it can lead to fuzzy image edge details, reducing noise removal performance. Wiener filtering recovery to protect the edges of the image is poor. So the wiener filtering recovery is not good at dealing with the convokernellution kernel image blur and Gauss noise pollution. But the image restoration using the TV model has the dual effect of denoising and to blur. In short, the recovery image and the original image are put in a very big difference using the other two methods, and TV model is compared with the original image that is close to the results of recovery.

\section{CONCLUSION}

In this paper, an improved total variation model of image restoration is presented according to the characteristics of the microscopic images of CHM. The microscopic images of CHM are blurred using the two dimensional different Gaussian convolution kernels and contaminated by the different Gauss white noise; the damaged images are restored by utilizing the improved TV model. Image restoration technology of TV model is a kind of ideal recovery method, which provides a good reference for image restoration following the image processing.

\section{CONFLICT OF INTEREST}

The authors confirm that this article content has no conflict of interest.

\section{ACKNOWLEDGEMENTS}

This work is supported by National Natural Science Foundation of P. R. China (61461046, 81260525), Higher
School in Gansu Province Basic Scientific Research Expenses (2050205), Natural Science Foundation of Gansu Province (1010RJZE028) and Tianshui youth scientific and technological support projects (TSK1201).

\section{REFERENCES}

[1] Y.J. Zhou, and B.R. Xiang, "Chinese medicine method of identifying research progress," Strait Pharmaceutical Journal, vol. 21, pp. 20-23, 2009.

[2] W. Lu, X.Y. Qin, W. Chen, and S.T. Chen, "Automatic hotspots recognition and trends prediction in traditional medicine," In: Proceedings of IEEE International Conference on IT in Medicine \& Education (ITME), Xiamen, China, 2008, pp. 12-14.

[3] H.Y. Liu, Z.H. Wei, and Z.R. Zhang, "Adaptive nonlocal patch regularization for image restoration," Acta Electronica Sinica, vol. 40, pp. 512-517, 2012.

[4] Z.F. Zhou, and P.L. Shui, "Blind image restoration algorithm iteratively using wavelet denoising and total variation regularization," Journal of Electronics \& Information Technology, vol. 30, pp. 2912-2915, 2008.

[5] J. Cheng, C. Chun, and J.J. Bu, "A new approach of blind image restoration," In: Proceedings of IEEE International Conference on Systems, Man \& Cybernetics, Washington, D.C: USA, 2003, pp. 58.

[6] F.F. Meng, and S.Y. Shang, "Fuzzy fiber image restoration based on blind deconvolution algorithm," Journal of Beijing Institute of Clothing Technology, vol. 33, pp. 53-58, 2011.

[7] N. Weiner. Extrapolation, Interpolation, and Smoothing of Stationary Time Series, The MIT Press: Cambridge, Mass, 1942.

[8] L. Rudin, S. Osher, and E. Fatime, "Nonlinear total variation bases noise removal algorithm," Physisca D, vol. 60, pp. 259-268, 1992.

[9] Y.L. Lin, J.H Zhao, and X.F. Zhu, "Improved algorithm based on TV model for image inpainting," Computer Engineering and Design, vol. 31, pp. 776-779, 2010. 